\title{
Generic Well-posedness for an Inverse Source Problem for a Multi-term Time-fractional Diffusion Equation
}

\author{
Zhiyuan Li*, Xing Cheng and Yikan Liu
}

\begin{abstract}
This paper deals with an inverse source problem for the multi-term timefractional diffusion equation with a diffusion parameter by using final overdetermination. On the basis of analytic Fredholm theory, a generic well-posedness of the inverse source problem in some suitable function space is proved.
\end{abstract}

\section{Introduction and main result}

In recent years, more and more anomalous diffusion processes whose mean square displacement (MSD) is of the power type $C_{\alpha} t^{\alpha}(0<\alpha<1)$, as $t \rightarrow \infty$, were found in many fields: biology, polymer physics, chemistry, biochemistry and etc. As is known, the MSD of the processes modeled by the classical diffusion equation behaviors like $C t$, as $t \rightarrow \infty$, so the classical diffusion models are inadequate to model these anomalous diffusion. On the other hand, these anomalous diffusion processes usually admit a non-Gaussian profile in space which are poorly characterized by the classical diffusion model, for example, [1] pointed out that the field data in the saturated zone of a highly heterogeneous aquifer indicated the long-tailed profile in the spatial distribution of densities as the time passes, which cannot be described by Gaussian processes, see, e.g., [5, 11] and the references therein. In many attempts for modeling the anomalous diffusion processes mathematically, there is an effective one named time-fractional diffusion equation (TFDE) which is obtained by replacing the first-order time derivative by a fractional derivative of order $\alpha \in(0,1)$, that is,

$$
\partial_{t}^{\alpha} u-\Delta u=F, \quad(x, t) \in \mathbb{R}^{d} \times(0, \infty) .
$$

Here by $\partial_{t}^{\alpha}$, we denote the Caputo fractional derivative with respect to $t$ :

$$
\partial_{t}^{\alpha} g(t)=\frac{1}{\Gamma(1-\alpha)} \int_{0}^{t}(t-\tau)^{-\alpha} \frac{d}{d \tau} g(\tau) d \tau
$$

Received November 19, 2018; Accepted November 5, 2019.

Communicated by Eiji Yanagida.

2010 Mathematics Subject Classification. Primary: 35R30; Secondary: 35R25, 47A55.

Key words and phrases. multi-term time-fractional diffusion equation, inverse source problem, Fredholm alternative.

*Corresponding author. 
and $\Gamma(\cdot)$ is the Gamma function. See, e.g., 25 for the definition and properties of Caputo derivative. This modified model is presented as a useful approach for the description of transport dynamics in complex system that are governed by anomalous diffusion and non-exponential relaxation patterns, and attracted great attention from different areas. For example, the TFDEs well captured the slow decay in time and non-Gaussian profile in space for the diffusion in the heterogeneous anomalous medium, see, e.g., [5, 11] and the references therein. For the theoretics, we refer to $3,10,21,22,26]$ and the references therein. Regarding numerical treatments, we refer to $9,15,19,24$.

As a natural extension, the multi-term time-fractional diffusion equation (MTTFDE) with a linear combination of fractional derivatives is proposed, which is expected to improve the modeling accuracy in depicting the anomalous diffusion due to its potential feasibility. Regarding physical and practical importance, in this paper, we consider the following initial-boundary value problem for the MTTFDE

$$
\begin{cases}\sum_{j=1}^{\ell} q_{j}(x) \partial_{t}^{\alpha_{j}} u+r A u+B(x) \cdot \nabla u=F & \text { in } \Omega \times(0, T] \\ u=0 & \text { in } \Omega \times\{0\} \\ u=0 & \text { on } \partial \Omega \times(0, T]\end{cases}
$$

where we assume $\Omega$ is a bounded domain in $\mathbb{R}^{d}$ with sufficiently smooth boundary $\partial \Omega$, and $0<\alpha_{\ell}<\cdots<\alpha_{1}<1$. The operator $A$ denotes a second-order partial differential operator, $q_{j} \in W^{2, \infty}(\Omega), j=1, \ldots, \ell$ and $B \in\left(W^{2, \infty}(\Omega)\right)^{d}$. Moreover $r>0$ is a parameter, and $T>0$ is a fixed value.

Owing to its applicability in describing the anomalous diffusion phenomena in highly heterogeneous media, the MTTFDEs gained increasing popularity among mathematicians within the last few decades, see, e.g., [8, 12, 14, 17, 18,20,23] and the the references therein. The article [14] discusses the spatial one dimensional case with constant coefficients where also the spatial fractional derivative is considered, and establishes the formula of the solution. In the paper [8], a solution of the corresponding initial-boundary value problem was formally represented in form of the Fourier series via the multivariate Mittag-Leffler function introduced in 21]. 23] assumes that the coefficients of the time derivatives are positive variable to prove unique existence of the solution by the Fourier method of the separation of the variables as well as the maximum principle and related properties. Later, 20] obtained a strong type maximum principle by carrying out the fundamental solution argument. In [18] the authors assert that the multi-term fractional diffusion equation is completely different from the classical diffusion case in terms of asymptotic behavior. More precisely, it was shown in [18] that the decay rate of the solutions $u$ to these problems was dominated by $t^{-\alpha_{\ell}}$ as $t \rightarrow \infty$, while $\|u(\cdot, t)\|_{H^{2}(\Omega)}$ is dominated by $t^{-\alpha_{1}}$ as $t \rightarrow 0$. In these papers, mainly the case of the symmetric fractional diffusion equations with the 
coefficients of lower order time-fractional derivatives being constants or positive variable were discussed. The case of symmetric fractional diffusion equations with the variable coefficients of time-fractional derivatives was recently considered in [4], where the regularity estimates of the solution were proved by constructing an integral equation corresponding to the initial-boundary value problem. [17] complemented the proof for the existence of the solution and further investigated several properties of the solution. Very recently, 12 established a Carleman type estimate of the solution by regarding the the lower fractional order term as a perturbation.

On the other hand, in many cases for the anomalous diffusion model such as (1.1), the term $f(x)$ models a source term, and it is often unknown and cannot be measured easily. However, these physical quantities can be identified from some additional information which can be observed or measured easily based on the forward problem. Here, assuming $F(x, t)=f(x) h(x, t)$, we consider the inverse source problem in determining spatial component $f$ from final overdetermination data. In other words, our main concern is

Inverse problem. Provided $h$ is known, we want to identify the spatial component $f$ from

$$
\varphi(x)=u(x, T), \quad x \in \Omega
$$

Throughout this paper, we assume

- $h \in C^{1}\left([0, T] ; L^{\infty}(\Omega)\right)$.

- There exists a constant $\delta>0$ satisfying $|h(x, T)| \geq \delta>0$ for $x \in \bar{\Omega}$.

We fix an arbitrary open interval $I \subset(0, \infty)$. Then for arbitrarily fixed $r \in I, f \in L^{2}(\Omega)$ and $h \in C^{1}\left([0, T] ; L^{\infty}(\Omega)\right)$, using the regularity of $h$ and modifying the proof in Lemma 2.4 in $\left[13\right.$, we can prove that there exists a unique solution $u[r, f] \in C\left([0, T] ; H^{2}(\Omega) \cap H_{0}^{1}(\Omega)\right)$ such that $\partial_{t}^{\alpha_{1}} u[r, f] \in C\left([0, T] ; L^{2}(\Omega)\right)$ and $(1.2)$. Moreover, there exists a constant $C>0$ such that

$$
\|u[r, f]\|_{C\left([0, T] ; H^{2}(\Omega)\right)}+\left\|\partial_{t}^{\alpha_{1}} u[r, f]\right\|_{C\left([0, T] ; L^{2}(\Omega)\right)} \leq C\|f\|_{L^{2}(\Omega)} .
$$

Here and henceforth in this section, $C$ denotes generic constants only depending on $r, d$, $\left\{\alpha_{j}\right\}_{j=1}^{\ell},\left\{q_{j}\right\}_{j=1}^{\ell}, \Omega, T$ and the coefficients of the operator $A$. Similar to the argument in 7,27$]$, we are ready to state our main theorem.

Theorem 1.1. We assume that $h \in C^{1}\left([0, T] ; L^{\infty}(\Omega)\right)$ satisfying $|h(x, T)|>\delta_{0}>0$ for a.e. $x \in \bar{\Omega}$. We fix an arbitrary open interval $I \subset(0, \infty)$. Then there exists a finite set $E=E\left(\alpha_{j}, h, I\right) \subset I$ satisfying: For $r \in I \backslash E$ and $\varphi \in H^{2}(\Omega) \cap H_{0}^{1}(\Omega)$, the problem (2.1) 
with (1.2) admits a unique solution $\{u, f\} \in C\left([0, T] ; H^{2}(\Omega) \cap H_{0}^{1}(\Omega)\right) \times L^{2}(\Omega)$. Moreover, there exists a constant $C=C\left(r, T, d, \alpha_{j}, \Omega, q_{j}, A\right)$ such that

$$
\|f\|_{L^{2}(\Omega)}+\|u[r, f]\|_{C\left([0, T] ; H^{2}(\Omega)\right)}+\left\|\partial_{t}^{\alpha_{1}} u[r, f]\right\|_{C\left([0, T] ; L^{2}(\Omega)\right)} \leq C\|\varphi\|_{L^{2}(\Omega)} .
$$

Remark 1.2. The theorem asserts that the well-posedness of our inverse source problem in the sense of Hadamard holds generically for diffusion constants.

\section{Proof of the main theorem}

In this section, we will set up notations, review some of standard facts on the fractional calculus, and prove Theorem 1.1. Let $L^{2}(\Omega)$ be a usual $L^{2}$-space with the inner product $(\cdot, \cdot)$ and $H^{1}(\Omega), H_{0}^{1}(\Omega)$ denote the Sobolev spaces (see, e.g., [2]). Without loss of generality, we assume $\ell=2$ and consider the following initial-boundary value problem

$$
\begin{cases}\partial_{t}^{\alpha_{1}} u+q \partial_{t}^{\alpha_{2}} u+r A u+B \cdot \nabla u=f(x) h(x, t), & (x, t) \in \Omega \times(0, T], \\ u(x, 0)=0, & x \in \Omega, \\ u(x, t)=0, & (x, t) \in \partial \Omega \times(0, T] .\end{cases}
$$

We specify the second order elliptic operator $A$ as follows:

$$
A u=-\sum_{i, j=1}^{d} \partial_{i}\left(a_{i j} \partial_{j} u\right), \quad u \in D(A):=H^{2}(\Omega) \cap H_{0}^{1}(\Omega),
$$

where $a_{i j}=a_{j i} \in C^{1}(\bar{\Omega}), 1 \leq i, j \leq d$, and there exists a constant $a_{0}>0$ such that

$$
a_{0} \sum_{j=1}^{d} \xi_{j}^{2} \leq \sum_{j, k=1}^{d} a_{j k}(x) \xi_{j} \xi_{k}, \quad x \in \bar{\Omega}, \xi \in \mathbb{R}^{d} .
$$

Since $A$ is a symmetric uniformly elliptic operator, we see that the Dirichlet eigensystem $\left\{\lambda_{n}, \phi_{n}\right\}$ of $A$ satisfies $0<\lambda_{1}<\lambda_{2} \leq \cdots \leq \lambda_{n} \leq \cdots$, and the sequence $\left\{\phi_{n}\right\}_{n=1}^{\infty} \subset D(A)$ is an orthonormal basis in $L^{2}(\Omega)$. Then the fractional power $A^{\gamma}$ for $\gamma \in \mathbb{R}$ can be defined by

$$
A^{\gamma} u:=\sum_{n=1}^{\infty} \lambda_{n}^{\gamma}\left(u, \phi_{n}\right) \phi_{n}, \quad u \in D\left(A^{\gamma}\right):=\left\{\psi \in L^{2}(\Omega) ; \sum_{n=1}^{\infty} \lambda_{n}^{2 \gamma}\left|\left(\psi, \phi_{n}\right)\right|^{2}<\infty\right\} .
$$

Moreover, $D\left(A^{\gamma}\right)$ is a Hilbert space with the norm $\|\psi\|_{D\left(A^{\gamma}\right)}^{2}=\sum_{n=1}^{\infty} \lambda_{n}^{2 \gamma}\left|\left(\psi, \phi_{n}\right)\right|^{2}$. For any $r \in \mathcal{S}:=\{z \in \mathbb{C} ; \mu \leq|\arg z| \leq \pi\}$ with $\mu \in\left(\frac{\pi}{2} \alpha_{1}, \pi \alpha_{1}\right)$, we define an operator $S_{r}(t): L^{2}(\Omega) \rightarrow L^{2}(\Omega), t>0$ by

$$
S_{r}(t) a:=\sum_{n=1}^{\infty}\left(a, \phi_{n}\right) E_{\alpha_{1}, 1}\left(-\lambda_{n} r t^{\alpha_{1}}\right) \phi_{n}, \quad a \in L^{2}(\Omega)
$$


where $E_{\alpha, \beta}(\cdot), \alpha, \beta>0$, is the Mittag-Leffler function defined by

$$
E_{\alpha, \beta}(z):=\sum_{k=0}^{\infty} \frac{z^{k}}{\Gamma(\alpha k+\beta)}, \quad z \in \mathbb{C} .
$$

It is known from, e.g., Theorem 1.6 in 25] that term-wise differentiations with respect to $t$ in the definition of $S_{r}(t)$ are possible and give

$$
\begin{aligned}
\frac{d S_{r}(t) a}{d t} & =-r \sum_{n=1}^{\infty} \lambda_{n}\left(a, \phi_{n}\right) t^{\alpha_{1}-1} E_{\alpha_{1}, \alpha_{1}}\left(-\lambda_{n} r t^{\alpha_{1}}\right) \phi_{n}, \\
\frac{d^{2} S_{r}(t) a}{d t^{2}} & =-r \sum_{n=1}^{\infty} \lambda_{n}\left(a, \phi_{n}\right) t^{\alpha_{1}-2} E_{\alpha_{1}, \alpha_{1}-1}\left(-\lambda_{n} r t^{\alpha_{1}}\right) \phi_{n}, \quad a \in L^{2}(\Omega),
\end{aligned}
$$

where $t>0$ and $r \in \mathcal{S}$. For later use, we also adopt the abbreviation

$$
S_{r}^{\prime}(t) a:=S_{r}^{(1)}(t) a:=\frac{d S_{r}(t) a}{d t}, \quad S_{r}^{\prime \prime}(t) a:=S_{r}^{(2)}(t) a:=\frac{d^{2} S_{r}(t) a}{d t^{2}} .
$$

Lemma 2.1. Let $r \in \mathcal{S}$ and $0<t<T$. Concerning the above defined operator $S_{r}(t)$, the following inequalities hold true.

(a) there exists a constant $C>0$ such that

$$
\left\|S_{r}(t)\right\|_{L^{2}(\Omega) \rightarrow L^{2}(\Omega)} \leq C|r|^{-1} t^{-\alpha_{1}}
$$

(b) for $0 \leq \gamma<1$, there exists a constant $C>0$ such that

$$
\left\|A^{\gamma} S_{r}(t)\right\|_{L^{2}(\Omega) \rightarrow L^{2}(\Omega)} \leq C|r|^{-\gamma} t^{-\alpha_{1} \gamma}
$$

(c) for $0 \leq \gamma \leq 1$, there exists a constant $C>0$ such that

$$
\left\|A^{\gamma-1} S_{r}^{(k)}(t)\right\|_{L^{2}(\Omega) \rightarrow L^{2}(\Omega)} \leq C|r|^{1-\gamma} t^{\alpha_{1}(1-\gamma)-k}, \quad k=1,2,
$$

where $\|\cdot\|_{L^{2}(\Omega) \rightarrow L^{2}(\Omega)}$ denotes the operator norm from $L^{2}(\Omega)$ to $L^{2}(\Omega)$.

Proof. First, to prove (a), from the definition of the operator $S_{r}(t)$, it follows that

$$
S_{r}(t) a=\sum_{n=1}^{\infty}\left(a, \phi_{n}\right) E_{\alpha_{1}, 1}\left(-\lambda_{n} r t^{\alpha_{1}}\right) \phi_{n}
$$

and so

$$
\left\|S_{r}(t) a\right\|_{L^{2}(\Omega)}^{2}=\sum_{n=1}^{\infty}\left|\left(a, \phi_{n}\right)\right|^{2}\left|E_{\alpha_{1}, 1}\left(-\lambda_{n} r t^{\alpha_{1}}\right)\right|^{2} \leq \sum_{n=1}^{\infty}\left|\left(a, \phi_{n}\right)\right|^{2}\left(\frac{C}{1+\lambda_{n} r t^{\alpha_{1}}}\right)^{2} .
$$


Here in the equation we used the Parseval equality, in the inequality we used

$$
\left|E_{\alpha_{1}, 1}\left(-\lambda_{n} r t^{\alpha_{1}}\right)\right| \leq \frac{C}{1+\lambda_{n}|r| t^{\alpha_{1}}}
$$

if $\mu<\arg r<\pi$ (e.g., 25, Theorem 1.6, p. 35]). Therefore

$$
\left\|S_{r}(t) a\right\|_{L^{2}(\Omega)}^{2} \leq C \sum_{n=1}^{\infty}\left|\left(a, \phi_{n}\right)\right|^{2}\left(\frac{1}{\lambda_{n}|r| t^{\alpha_{1}}}\right)^{2} \leq C \lambda_{1}^{-2}|r|^{-2} t^{-2 \alpha_{1}}\|a\|_{L^{2}(\Omega)},
$$

which proves 2.2 .

Next we prove (b). For $\gamma \in[0,1)$, again from the definition of the operator $S_{r}(t)$, it follows that

$$
A^{\gamma} S_{r}(t) a=\sum_{n=1}^{\infty} \lambda_{n}^{\gamma}\left(a, \phi_{n}\right) E_{\alpha_{1}, 1}\left(-\lambda_{n} r t^{\alpha_{1}}\right) \phi_{n},
$$

and so by an argument similar to the above conclusion we have

$$
\begin{aligned}
\left\|A^{\gamma} S_{r}(t) a\right\|_{L^{2}(\Omega)}^{2} & =\sum_{n=1}^{\infty} \lambda_{n}^{2 \gamma}\left|\left(a, \phi_{n}\right)\right|^{2}\left|E_{\alpha_{1}, 1}\left(-\lambda_{n} r t^{\alpha_{1}}\right)\right|^{2} \\
& \leq \sum_{n=1}^{\infty} \lambda_{n}^{2 \gamma}\left|\left(a, \phi_{n}\right)\right|^{2}\left(\frac{1}{1+\lambda_{n} r t^{\alpha_{1}}}\right)^{2}
\end{aligned}
$$

if $\mu<\arg r<\pi$. Therefore

$$
\begin{aligned}
\left\|A^{\gamma} S_{r}(t) a\right\|_{L^{2}(\Omega)}^{2} & \leq C r^{-2 \gamma} t^{-2 \alpha_{1} \gamma} \sum_{n=1}^{\infty}\left|\left(a, \phi_{n}\right)\right|^{2}\left(\frac{\lambda_{n}^{\gamma}|r|^{\gamma} t^{\alpha_{1} \gamma}}{1+\lambda_{n}|r| t^{\alpha_{1}}}\right)^{2} \\
& \leq C|r|^{-2 \gamma} t^{-2 \alpha_{1} \gamma} \max _{\eta \geq 0}\left(\frac{\eta^{\gamma}}{1+\eta}\right)^{2} \sum_{n=1}^{\infty}\left|\left(a, \phi_{n}\right)\right|^{2},
\end{aligned}
$$

which proves 2.3 . Here we note that $\max _{\eta \geq 0} \frac{\eta^{\gamma}}{1+\eta}<\infty$ by $0 \leq \gamma \leq 1$.

Estimating $A^{\gamma-1} S_{r}^{(k)}(t)(k=1,2)$ similarly, in fact, for $\gamma \in[0,1]$, we have

$$
A^{\gamma-1} S_{r}^{(k)}(t) a=-r \sum_{n=1}^{\infty} \lambda_{n}^{\gamma}\left(a, \phi_{n}\right) t^{\alpha_{1}-k} E_{\alpha_{1}, \alpha_{1}-k+1}\left(-\lambda_{n} r t^{\alpha_{1}}\right) \phi_{n}
$$

and so

$$
\begin{aligned}
\left\|A^{\gamma-1} S_{r}^{(k)}(t) a\right\|_{L^{2}(\Omega)}^{2} & =r^{2} t^{2\left(\alpha_{1}-k\right)} \sum_{n=1}^{\infty} \lambda_{n}^{2 \gamma}\left|\left(a, \phi_{n}\right)\right|^{2}\left|E_{\alpha_{1}, \alpha_{1}-k+1}\left(-\lambda_{n} r t^{\alpha_{1}}\right)\right|^{2} \\
& \leq C r^{2} t^{2\left(\alpha_{1}-k\right)} \sum_{n=1}^{\infty} \lambda_{n}^{2 \gamma}\left|\left(a, \phi_{n}\right)\right|^{2}\left(\frac{1}{1+\lambda_{n} r t^{\alpha_{1}}}\right)^{2} \\
& \leq C|r|^{2(1-\gamma)} t^{2\left(\alpha_{1}-k-\alpha_{1} \gamma\right)} \max _{\eta \geq 0}\left(\frac{\eta^{\gamma}}{1+\eta}\right)^{2} \sum_{n=1}^{\infty}\left|\left(a, \phi_{n}\right)\right|^{2}
\end{aligned}
$$

which proves 2.4 . 
For any fixed $T>0$, we define an operator $A_{r}$ by

$$
A_{r} f:=\frac{\partial_{t}^{\alpha_{1}} u[r, f](\cdot, T)}{h(\cdot, T)}+\frac{q \partial_{t}^{\alpha_{2}} u[r, f](\cdot, T)}{h(\cdot, T)},
$$

where the function $u[r, f]$ is the unique solution to the initial-boundary value problem (2.1). The above definition of $A_{r}$ is well-posed since the problem (2.1) admits a unique solution $u[r, f] \in C\left([0, T] ; H^{2}(\Omega) \cap H_{0}^{1}(\Omega)\right)$ such that $\partial_{t}^{\alpha_{1}} u[r, f] \in C\left([0, T] ; L^{2}(\Omega)\right)$ provided that $h \in C^{1}\left([0, T] ; L^{\infty}(\Omega)\right)$. Then we can rephrase the problem (2.1) into the following equation

$$
A_{r} f+\Phi=f, \quad \Phi:=\frac{r A \varphi+B \cdot \nabla \varphi}{h(\cdot, T)},
$$

where $f$ is unknown.

Lemma 2.2. Let $r \in I$ be arbitrary fixed, we see that (2.5) possesses a unique solution if and only if there exists a unique solution $\{u[r, f], f\}$ to [2.1] and (1.2).

Proof. First, we assume that (2.5) possesses a unique solution $f \in L^{2}(\Omega)$. Substituting the solution $f$ into 2.1 , then we have a unique solution $u[r, f] \in C\left([0, T] ; H^{2}(\Omega)\right)$. It is not difficult to prove that that the solution $u[r, f]$ satisfies the observation data $u[r, f](T)=\varphi$. For this, we set

$$
\varphi_{1}(x):=u[r, f](x, T), \quad x \in \Omega .
$$

Then we can see that $\varphi_{1} \in H^{2}(\Omega) \cap H_{0}^{1}(\Omega)$. From $(2.5)$, we have

$$
\frac{1}{h(\cdot, T)}(r A+B \cdot \nabla)\left(\varphi-\varphi_{1}\right)=0 \quad \text { in } \Omega \text {. }
$$

Since $\left.\left(\varphi-\varphi_{1}\right)\right|_{\partial \Omega}=0$, by Maximum principle for the weak solution (e.g., 6, Theorem 1.35, p. 22]) we have

$$
\varphi(x)=\varphi_{1}(x), \quad x \in \bar{\Omega} .
$$

Now we suppose that both $\left\{u\left[r, f_{1}\right], f_{1}\right\}$ and $\left\{u\left[r, f_{2}\right], f_{2}\right\}$ are two solutions to (2.1) and satisfies the same observation data 1.2 . Then we must have $f_{1}=f_{2}$. If not, we see that both $f_{1}$ and $f_{2}$ are solutions to (2.5). Consequently, $u\left[r, f_{1}\right]=u\left[r, f_{2}\right]$ because of the unique solvability of the direct problem 2.1.

Conversely, we assume that the solution $\{u[r, f], f\}$ to $(2.1)$ and $(1.2)$ exists uniquely. If $f_{1}$ and $f_{2}$ are distinct solutions to (2.5), then there exist solutions $\left\{u\left[r, f_{1}\right], f_{1}\right\}$ and $\left\{u\left[r, f_{2}\right], f_{2}\right\}$ to $(2.1)$ and $(1.2)$, which contradicts the unique solvability to (2.1) and (1.2). Thus the proof of Lemma 2.2 is completed.

The solvability of the equation (2.5) can be done by using the analytic Fredholm alternative from the operator theory. For this treatment, we need establish several lemmas. 
Lemma 2.3. $A_{r}: L^{2}(\Omega) \rightarrow L^{2}(\Omega)$ is compact operator for any $r>0$.

Proof. Since $u:=u[r, f]$ is the solution of the initial-boundary value problem (2.1), by an argument similar to the proof of Theorem 1 in [4], for any $0<t \leq T$ and $r>0$, we find

$$
\begin{aligned}
A u(t)= & r^{-1} \int_{0}^{t} A^{1 / 2-1} S_{r}^{\prime}(t-\tau)\left(A^{1 / 2}(B \cdot \nabla u)\right) d \tau-r^{-1} \int_{0}^{t} S_{r}^{\prime}(t-\tau)(f h(\cdot, \tau)) d \tau \\
& +\frac{r^{-1}}{\Gamma\left(1-\alpha_{2}\right)} \int_{0}^{t} d \eta \int_{0}^{t-\tau}\left(\eta^{-\alpha_{2}}-(t-\tau)^{-\alpha_{2}}\right) A^{-1} S_{r}^{\prime \prime}(t-\eta-\tau)(A(q u)) d \tau \\
& -\frac{r^{-1}}{\Gamma\left(1-\alpha_{2}\right)} \int_{0}^{t}(t-\tau)^{-\alpha_{2}} A^{-1} S_{r}^{\prime}(t-\tau)(A(q u)) d \tau=: r^{-1} \sum_{i=1}^{4} I_{i}(t ; r, f) .
\end{aligned}
$$

We define $\left(\mathcal{B}_{r} f\right)(x):=h(x, T)\left(A_{r} f\right)(x)$, and we will prove $\mathcal{B}_{r}: L^{2}(\Omega) \rightarrow L^{2}(\Omega)$ is a compact operator for any $r>0$. From the definition of $A_{r}$ and noting (2.5), we have

$$
\left(\mathcal{B}_{r} f\right)(x)=-\sum_{i=1}^{4} I_{i}(T ; r, f)-B \cdot \nabla u(r, f)(T)+f(x) h(x, T) .
$$

Since $h \in C^{1}\left([0, T] ; L^{2}(\Omega)\right)$, integrating by part, we obtain

$$
\begin{aligned}
\int_{0}^{T} S_{r}^{\prime}(T-\tau)(f h(\cdot, \tau)) d \tau= & -\left.S_{r}(T-\tau)(f h(\cdot, \tau))\right|_{\tau=0} ^{\tau=T} \\
& +\int_{0}^{T} S_{r}(T-\tau)\left(f h_{\tau}(\cdot, \tau)\right) d \tau
\end{aligned}
$$

Therefore

$$
\begin{aligned}
\mathcal{B}_{r} f= & -I_{1}(T ; r, f)-I_{3}(T ; r, f)-I_{4}(T ; r, f)-B \cdot \nabla u(\cdot, T) \\
& +S_{r}(T)(f h(\cdot, 0))+\int_{0}^{T} S_{r}(T-\tau)\left(f h_{\tau}(\cdot, \tau)\right) d \tau .
\end{aligned}
$$

Now choosing $\varepsilon>0$ small enough such that $\varepsilon<1 / 2$ and $\alpha_{1}-\alpha_{2}-\alpha_{1} \varepsilon>0$, from (2.4) and $(1.3)$, we see that

$$
\begin{aligned}
\left\|I_{1}(T ; r, f)\right\|_{D\left(A^{\varepsilon}\right)} & =\left\|A^{\varepsilon} I_{1}(T ; r, f)\right\|_{L^{2}(\Omega)} \leq\left\|\int_{0}^{T} A^{\varepsilon-1 / 2} S_{r}^{\prime}(T-\tau) A^{1 / 2}(B \cdot \nabla u) d \tau\right\|_{L^{2}(\Omega)} \\
& \leq C|r|^{1 / 2-\varepsilon} \int_{0}^{T}(T-\tau)^{\alpha_{1}(1 / 2-\varepsilon)-1}\|u\|_{D(A)} d \tau \leq C_{r}\|f\|_{L^{2}(\Omega)},
\end{aligned}
$$

and

$$
\begin{aligned}
\left\|I_{4}(T ; r, f)\right\|_{D\left(A^{\varepsilon}\right)} & \leq\left\|\int_{0}^{T} A^{\varepsilon-1} S_{r}^{\prime}(T-\tau)(T-\tau)^{-\alpha_{2}} A(q u) d \tau\right\|_{L^{2}(\Omega)} \\
& \leq C|r|^{1-\varepsilon} \int_{0}^{T}(T-\tau)^{\alpha_{1}(1-\varepsilon)-1-\alpha_{2}}\|A u(r)\|_{L^{2}(\Omega)} d \tau \leq C_{r}\|f\|_{L^{2}(\Omega)} .
\end{aligned}
$$


Similarly, we can prove that

$$
\begin{gathered}
\|B \cdot \nabla u\|_{D\left(A^{\varepsilon}\right)} \leq C_{r}\|f\|_{L^{2}(\Omega)}, \\
\left\|S_{r}(T)(f h(\cdot, T))\right\|_{D\left(A^{\varepsilon}\right)} \leq C|r|^{-\varepsilon}\|f\|_{L^{2}(\Omega)},
\end{gathered}
$$

and

$$
\left\|\int_{0}^{T} S_{r}(T-\tau)\left(f h_{\tau}(\cdot, \tau)\right) d \tau\right\|_{D\left(A^{\varepsilon}\right)} \leq C|r|^{-\varepsilon}\|f\|_{L^{2}(\Omega)} .
$$

To evaluate $A^{\varepsilon} I_{3}(T ; r, f)$ we need more technical treatment to the integral in $I_{3}(T ; r, f)$. In fact, after the change of variable $\widetilde{\tau}=T-\tau$, and letting $\widetilde{\eta}=\eta / \widetilde{\tau}$, we use (2.4) and (1.3) to derive that

$$
\begin{aligned}
& \left\|I_{3}(T ; r, f)\right\|_{D\left(A^{\varepsilon}\right)} \\
\leq & C_{r} \int_{0}^{T}\|u(T-\widetilde{\tau})\|_{D(A)} d \widetilde{\tau} \int_{0}^{1}(\widetilde{\tau}-\widetilde{\tau} \widetilde{\eta})^{\alpha_{1}(1-\varepsilon)-2}\left((\widetilde{\tau} \widetilde{\eta})^{-\alpha_{2}}-\widetilde{\tau}^{-\alpha_{2}}\right) \widetilde{\tau} d \widetilde{\eta} \\
= & C_{r} \int_{0}^{T}\left[\int_{0}^{1}(1-\eta)^{\alpha_{1}(1-\varepsilon)-2}\left(\eta^{-\alpha_{2}}-1\right) d \eta\right] \tau^{\alpha_{1}(1-\varepsilon)-\alpha_{2}-1}\|f\|_{L^{2}(\Omega)} d \tau .
\end{aligned}
$$

Finally, we prove $J:=\int_{0}^{1}(1-\eta)^{\alpha_{1}(1-\varepsilon)-2}\left(\eta^{-\alpha_{2}}-1\right) d \eta<\infty$. In fact, we represent $J$ in the form

$$
J=\int_{0}^{1 / 2}(1-\eta)^{\alpha_{1}(1-\varepsilon)-2}\left(\eta^{-\alpha_{2}}-1\right) d \eta+\int_{1 / 2}^{1}(1-\eta)^{\alpha_{1}(1-\varepsilon)-2}\left(\eta^{-\alpha_{2}}-1\right) d \eta:=J^{\prime}+J^{\prime \prime} .
$$

For $J^{\prime}$, using the inequality $(1-\eta)^{\alpha_{1}(1-\varepsilon)-2} \leq\left(\frac{1}{2}\right)^{\alpha_{1}(1-\varepsilon)-2}, \forall \eta \in[0,1 / 2]$, we derive

$$
J^{\prime} \leq C \int_{0}^{1 / 2}\left(\eta^{-\alpha_{2}}-1\right) d \eta<\infty .
$$

For $J^{\prime \prime}$, using the inequality $\eta^{-\alpha_{2}}-1 \leq C(1-\eta) \eta^{-\alpha_{2}-1}, \forall \eta \in(0,1)$, which can be proved, for example, by means of the mean value theorem, we can deduce

$$
J^{\prime \prime} \leq C \int_{1 / 2}^{1}(1-\eta)^{\alpha_{1}(1-\varepsilon)-2}(1-\eta) \eta^{-\alpha_{2}-1} d \eta=C \int_{1 / 2}^{1}(1-\eta)^{\alpha_{1}(1-\varepsilon)-1} \eta^{-\alpha_{2}-1} d \eta<\infty .
$$

Collecting the above estimates, we have

$$
\left\|\mathcal{B}_{r} f\right\|_{H^{2 \varepsilon}(\Omega)} \leq C\left\|\mathcal{B}_{r} f\right\|_{D\left(A^{\varepsilon}\right)} \leq C_{r}\|f\|_{L^{2}(\Omega)} .
$$

Since the Sobolev space $H^{2 \varepsilon}(\Omega)$ is compactly embedded into $L^{2}(\Omega)$, we obtain for any fixed $r>0$, the operator $\mathcal{B}_{r}: L^{2}(\Omega) \rightarrow L^{2}(\Omega)$ is compact. Furthermore, since $h(x, T) \geq \delta>0$, for $x \in \bar{\Omega}$, the division operator by $h(\cdot, T)$ is bounded from $L^{2}(\Omega)$ to $L^{2}(\Omega)$. As a result, $A_{r}: L^{2}(\Omega) \rightarrow L^{2}(\Omega)$ is compacted for $\forall r>0$. Thus the proof of Lemma 2.3 is completed. 
Lemma 2.4. There exists a large $R^{*}$ such that for any $r>R^{*}$, there exists a constant $0<\theta(r)<1$ satisfying

$$
\left\|A_{r} f\right\|_{L^{2}(\Omega)} \leq \theta(r)\|f\|_{L^{2}(\Omega)} .
$$

Proof. First, from (2.2) and (2.7), for any $0<t \leq T$ we have

$$
\begin{aligned}
\left\|\int_{0}^{t} S_{r}^{\prime}(t-\tau)\left(f h_{\tau}(\cdot, \tau)\right) d \tau\right\|_{L^{2}(\Omega)} \leq & C\left(1+r^{-1} t^{-\alpha_{1}}\right)\|f\|_{L^{2}(\Omega)} \\
& +C \int_{0}^{t}(t-\tau)^{-\alpha_{1}} r^{-1}\|f\|_{L^{2}(\Omega)} d \tau .
\end{aligned}
$$

By the above inequality and (2.6), and a similar argument used in the proof of Lemma 2.3 . we can show that

$$
\begin{aligned}
\|u(t)\|_{D(A)} \leq & C r^{-1 / 2} \int_{0}^{t}(t-\tau)^{\alpha_{1} / 2-1}\left\|A^{1 / 2}(B \cdot \nabla u(\tau))\right\|_{L^{2}(\Omega)} d \tau \\
& +C\left(r^{-1}+r^{-2}+t^{-\alpha_{1}} r^{-2}\right)\|f\|_{L^{2}(\Omega)} \\
& +C r^{-1} \int_{0}^{t}(t-\tau)^{\alpha_{1}-\alpha_{2}-1} r\|A(q u(\tau))\|_{L^{2}(\Omega)} d \tau, \quad 0<t \leq T .
\end{aligned}
$$

Denoting $\bar{\alpha}:=\min \left\{\alpha_{1} / 2, \alpha_{1}-\alpha_{2}\right\}$, for $r>1$, we obtain

$$
\|u(t)\|_{D(A)} \leq C r^{-1} t^{-\alpha_{1}}\|f\|_{L^{2}(\Omega)}+C \int_{0}^{t}(t-\tau)^{\bar{\alpha}-1}\|u(\tau)\|_{D(A)} d \tau, \quad 0<t \leq T,
$$

which combined with the general Gronwall inequality (see, e.g., [25]) implies

$$
\|u(t)\|_{D(A)} \leq C r^{-1} t^{-\alpha_{1}}\|f\|_{L^{2}(\Omega)}, \quad 0<t \leq T .
$$

From 2.1], choosing $\varepsilon>0$ the same as that in Lemma 2.3, we find

$$
\begin{aligned}
& h(\cdot, T) A_{r} f \\
= & \int_{0}^{T} A^{-1 / 2} S_{r}^{\prime}(T-\tau)\left(A^{1 / 2}(B \cdot \nabla u)\right) d \tau+\int_{0}^{T} S_{r}(T-\tau)\left(f h_{\tau}(\cdot, \tau)\right) d \tau \\
& +S_{r}(T)(f h(\cdot, 0))+B \cdot \nabla u(\cdot, T) \\
& +\frac{1}{\Gamma\left(1-\alpha_{2}\right)} \int_{0}^{T}(T-\tau)^{-\alpha_{2}} A^{\varepsilon-1} S_{r}^{\prime}(T-\tau)\left(A^{1-\varepsilon}(q u)\right) d \tau \\
& -\frac{1}{\Gamma\left(1-\alpha_{2}\right)} \int_{0}^{T}\left(\eta^{-\alpha_{2}}-(T-\tau)^{-\alpha_{2}}\right) d \tau \int_{0}^{T-\tau} A^{\varepsilon-1} S_{r}^{\prime \prime}(T-\eta-\tau)\left(A^{1-\varepsilon}(q u)\right) d \eta .
\end{aligned}
$$

Using (2.2) and 2.4), similar to Lemma 2.3, we derive

$$
\begin{aligned}
\left\|A_{r} f\right\|_{L^{2}(\Omega)} \leq & C \int_{0}^{T}(T-\tau)^{\alpha_{1} / 2-1} r^{1 / 2}\|u(\tau)\|_{D(A)} d \tau+C \int_{0}^{T}(T-\tau)^{-\alpha_{1}} r^{-1}\|f\|_{L^{2}(\Omega)} d \tau \\
& +C T^{-\alpha_{1}} r^{-1}\|f\|_{L^{2}(\Omega)}+C \int_{0}^{T}(T-\tau)^{\alpha_{1}-\alpha_{2}-\alpha_{1} \varepsilon-1} r^{1-\varepsilon}\left\|A^{1-\varepsilon} u(\tau)\right\|_{L^{2}(\Omega)} d \tau .
\end{aligned}
$$


Noting that $\left\|A^{1-\varepsilon} u\right\|_{L^{2}(\Omega)} \leq C\|u\|_{D(A)}, 0<t \leq T$, the use of the above estimates and (2.8) leads to

$$
\left\|A_{r} f\right\|_{L^{2}(\Omega)} \leq C r^{-1 / 2}\|f\|_{L^{2}(\Omega)}+C r^{-1}\|f\|_{L^{2}(\Omega)}+C r^{-\varepsilon}\|f\|_{L^{2}(\Omega)} .
$$

Consequently, when $r>0$ large enough, we deduce $\theta(r):=C\left(r^{-1}+r^{-1 / 2}+r^{-\varepsilon}\right) \in(0,1)$. Thus the proof of Lemma 2.4 is completed.

Now noting that the Mittag-Leffler function is analytic on $\mathbb{C}$, by an argument similar to the proof of the above lemma, we see that the diffusion parameter $r>0$ in the integral equation (2.6) can be extended to the complex value $r \in \mathcal{S}:=\{z \in \mathbb{C} ; \mu \leq|\arg (-z)| \leq \pi\}$, where $\frac{\pi}{2} \alpha_{1}<\mu<\pi \alpha_{1}$. In fact, we set $u_{0}[r, f]=0$, and for $k \in \mathbb{N}$ and $r \in \mathcal{S}$ we inductively define $u_{k+1}[r, f](x, t)$ as follows:

$$
\begin{aligned}
& u_{k+1}[r, f](\cdot, t) \\
= & -r^{-1} \int_{0}^{t} A^{-1} S_{r}^{\prime}(t-\tau)\left(B \cdot \nabla u_{k}[r, f](\cdot, \tau)\right) d \tau-r^{-1} \int_{0}^{t} A^{-1} S_{r}^{\prime}(t-\tau)(f h(\cdot, \tau)) d \tau \\
& +\frac{r^{-1}}{\Gamma\left(1-\alpha_{2}\right)} \int_{0}^{t}\left(\eta^{-\alpha_{2}}-(t-\tau)^{-\alpha_{2}}\right) d \tau \int_{0}^{t-\tau} A^{-1} S_{r}^{\prime \prime}(t-\eta-\tau)\left(q u_{k}[r, f](\cdot, \tau)\right) d \eta \\
& -\frac{r^{-1}}{\Gamma\left(1-\alpha_{2}\right)} \int_{0}^{t} A^{-1} S_{r}^{\prime}(t-\tau)(t-\tau)^{-\alpha_{2}}\left(q u_{k}[r, f](\cdot, \tau)\right) d \tau, \quad 0<t \leq T .
\end{aligned}
$$

For any $f \in L^{2}(\Omega)$ and any fixed $T>0$, we claim that

$$
\lim _{k \rightarrow \infty} u_{k}[r, f] \text { is analytic with respect to } r \in \mathcal{S} .
$$

More precisely, we have the analyticity of $A_{r} f$ with respect to the parameter $r \in \mathcal{S}$ presented in the following lemma.

Lemma 2.5. The operator $A_{r}: L^{2}(\Omega) \rightarrow L^{2}(\Omega)$ is analytic in $\mathcal{S}$ for any $t \in[0, T]$.

Proof. First, noting that the Mittag-Leffler function $E_{\alpha, \beta}(z)$ is analytic, it is easy to see that $A u_{k}[\cdot, f]: \mathcal{S} \rightarrow L^{2}(\Omega)$ is analytic. Next, we claim that for any compact set $K \subset \subset \mathcal{S}$, there exist constants $C_{1}, M$ only depending on $d, \Omega, T, K, q, \alpha_{1}, \alpha_{2}, B$ and the coefficients of the operator $A$ such that the following estimate

$$
\left\|\left(u_{k+1}[r, f]-u_{k}[r, f]\right)(\cdot, t)\right\|_{D(A)} \leq \frac{C_{1} M^{k} t^{k \bar{\alpha}}}{\Gamma(1+\bar{\alpha} k)}
$$

is valid for any $k \in \mathbb{N}, t \in[0, T], r \in K$. For simplicity, we let $u_{k}:=u_{k}[r, f], k \in \mathbb{N}$. We can prove this assertion by inductive argument. In fact, assuming that the above inequality holds for $k$, by (2.4), similarly to Lemma 2.3 , for any $t \in[0, T], r \in K$ we have

$$
\left\|\left(u_{k+1}[r, f]-u_{k}[r, f]\right)(\cdot, t)\right\|_{D(A)}
$$




$$
\begin{aligned}
\leq & \frac{C}{|r|^{1 / 2}} \int_{0}^{t}(t-\tau)^{\alpha_{1} / 2-1}\left\|B \cdot \nabla\left(u_{k}[r, f]-u_{k-1}[r, f]\right)(\cdot, \tau)\right\|_{D\left(A^{1 / 2}\right)} d \tau \\
& \left.+C|r| \int_{0}^{t}(t-\tau)^{\alpha_{1}-\alpha_{2}-1} \|\left(u_{k}[r, f]-u_{k-1}[r, f]\right)(\cdot, \tau)\right) \|_{D(A)} d \tau
\end{aligned}
$$

Letting $\bar{\alpha}:=\min \left(\alpha_{1} / 2, \alpha_{1}-\alpha_{2}\right)$, by induction assumption, and noting that the compact set $K \subset \subset \mathcal{S}$, we have $|r|^{-1 / 2}$ is bounded in $K$, and then

$$
\begin{aligned}
\left\|\left(u_{k+1}[r, f]-u_{k}[r, f]\right)(\cdot, t)\right\|_{D(A)} & \leq C_{K} \int_{0}^{t}(t-\tau)^{\bar{\alpha}-1}\left\|\left(u_{k}[r, f]-u_{k-1}[r, f]\right)(\cdot, \tau)\right\|_{D(A)} d \tau \\
& \leq C_{K} \int_{0}^{t}(t-\tau)^{\bar{\alpha}-1} \frac{C_{1} M^{k-1} \tau^{(k-1) \bar{\alpha}}}{\Gamma(1+\bar{\alpha}(k-1))} d \tau \\
& =\frac{C_{K} C_{1} M^{k-1}}{\Gamma(1+\bar{\alpha}(k-1))} \int_{0}^{t}(t-\tau)^{\widetilde{\alpha}-1} \tau^{(k-1) \bar{\alpha}} d \tau .
\end{aligned}
$$

Using $\frac{\Gamma(\alpha) \Gamma(\beta)}{\Gamma(\alpha+\beta)}=\int_{0}^{1} s^{\alpha-1}(1-s)^{\beta-1} d s, \alpha>0, \beta>0$, setting $M:=C_{K} \Gamma(\bar{\alpha})$, we find

$$
\left\|\left(u_{k+1}[r, f]-u_{k}[r, f]\right)(\cdot, t)\right\|_{D(A)} \leq \frac{C_{K} C_{1} M^{k-1} \Gamma(\bar{\alpha})}{\Gamma(\bar{\alpha} k+1)} t^{k \bar{\alpha}}=\frac{C_{1} M^{k} t^{k \bar{\alpha}}}{\Gamma(\bar{\alpha} k+1)}
$$

Therefore, for any compact set $K \subset \subset \mathcal{S}, A u_{k}[r, f]$ uniformly tends to a function $v[r, f]$ in $L^{2}(\Omega)$ as $k \rightarrow \infty$, hence that $v[r, f]$ is analytic in $\mathcal{S}$. Now we restrict the parameter back to the real number $r>0$. In this case, by uniqueness argument, we find $v[r, f]=A u[r, f]$, $r>0$, where $u[r, f]$ is the solution of the problem (2.1). Consequently, $A u[r, f]$ is also analytic in $\mathcal{S}$. Hence $A_{r}: L^{2}(\Omega) \rightarrow L^{2}(\Omega)$ is analytic in $\mathcal{S}$.

Proof of Theorem 1.1. From Lemmas 2.3 and 2.5, and the result on analytic perturbation to $A_{r}: L^{2}(\Omega) \rightarrow L^{2}(\Omega)$ (see, e.g., [16, Theorem 1.9, p. 370]), it follows that the following alternative holds:

(i) There exists a finite set $E=E\left(\alpha_{j}, h\right) \subset(0, \infty)$ such that 1 is not an eigenvalue of $A_{r}$ for all $r \in(0, \infty) \backslash E$.

(ii) 1 is an eigenvalue of $A_{r}$ for all $r \in(0, \infty)$.

Lemma 2.3 implies that 1 cannot be an eigenvalue of $A_{r}$ for large $r$, that is, (ii) cannot occur. Let $r \in I \backslash E$, we apply the Fredholm alternative to 2.5 to obtain

$$
\|f\|_{L^{2}(\Omega)} \leq C\|-r A \varphi\|_{L^{2}(\Omega)} \leq C\|\varphi\|_{H^{2}(\Omega)}
$$

which combined with (2.8) implies 1.4. The proof of the theorem is complete. 


\section{Concluding remarks}

In this paper, we considered an inverse source problem for multi-term time-fractional diffusion equations with a diffusion parameter $r>0$. This equation can be regarded as the perturbation of the classical governing fractional equation (i.e., $r=1$ ). By the MittagLeffler function and the eigenfunction expansion argument, we firstly gave an integral equation of the solution to the initial-boundary value problem, from which we verified the analyticity of the solution with respect to the diffusion parameter $r$. On the basis of the Fredholm alternative, we proved a generic stability result for our inverse source problem by the use of the analytic perturbation theory. Here we should mention that we do not know whether $I=\emptyset$. On the other hand, with an arbitrarily fixed $r>0$, e.g., $r=1$, we do not know whether there exists a unique pair $\{f, u\} \in L^{2}(\Omega) \times H^{2}(\Omega)$ satisfying the above initial-boundary value problem and the final observation data $u(x, T)=\varphi(x)$. Furthermore, in the proofs of our results, we need the assumption that all the coefficients are only $x$-dependent. It will be more interesting and challenging to consider what happens with the properties of the solutions in the case where the coefficients are both $t$ - and $x$ dependent.

\section{Acknowledgments}

The first and the third authors are partially supported by the A3 Foresight Program "Modeling and Computation of Applied Inverse Problems", Japan Society for the Promotion of Science (JSPS) and National Natural Science Foundation of China (NSFC). The first author is supported by NSFC (No. 11801326). The second author is supported by NSFC (No. 11526072). The third author is partially supported by JSPS KAKENHI Grant Number JP15H05740.

\section{References}

[1] E. E. Adams and L. W. Gelhar, Field study of dispersion in a heterogeneous aquifer: 2. Spatial moments analysis, Water Resources Research 28 (1992), no. 12, 3293-3307.

[2] R. A. Adams, Sobolev Spaces, Pure and Applied Mathematics 65, Academic Press, New York, 1975.

[3] E. G. Bajlekova, Fractional Evolution Equations in Banach Spaces, Dissertation, Technische Universiteit Eindhoven, Eindhoven, 2001.

[4] S. Beckers and M. Yamamoto, Regularity and unique existence of solution to linear diffusion equation with multiple time-fractional derivatives, in: Control and 
Optimization with PDE Constraints, 45-55, Internat. Ser. Numer. Math. 164, Birkhäuser/Springer Basel AG, Basel, 2013.

[5] J.-P. Bouchaud and A. Georges, Anomalous diffusion in disordered media: statistical mechanisms, models and physical applications, Phys. Rep. 195 (1990), no. 4-5, 127293.

[6] M. Choulli, Une introduction aux problèmes inverses elliptiques et paraboliques, Springer-Verlag, Berlin, 2009.

[7] M. Choulli and M. Yamamoto, Generic well-posedness of an inverse parabolic problem - the Hölder-space approach, Inverse Problems 12 (1996), no. 3, 195-205.

[8] V. Daftardar-Gejji and S. Bhalekar, Boundary value problems for multi-term fractional differential equations, J. Math. Anal. Appl. 345 (2008), no. 2, 754-765.

[9] K. Diethelm and Y. Luchko, Numerical solution of linear multi-term initial value problems of fractional order, J. Comput. Anal. Appl. 6 (2004), no. 3, 243-263.

[10] R. Gorenflo and F. Mainardi, Fractional calculus: integral and differential equations of fractional order, in: Fractals and Fractional Calculus in Continuum Mechanics (Udine, 1996), 223-276, CISM Courses and Lect. 378, Springer, Vienna, 1997.

[11] Y. Hatano and N. Hatano, Dispersive transport of ions in column experiments: An explanation of long-tailed profiles, Water Resources Research 34 (1998), no. 5, 10271033.

[12] X. Huang, Z. Li and M. Yamamoto, Carleman estimates for the time-fractional advection-diffusion equations and applications, Inverse Problems 35 (2019), no. 4, 045003, 36 pp.

[13] D. Jiang, Z. Li, Y. Liu and M. Yamamoto, Weak unique continuation property and a related inverse source problem for time-fractional diffusion-advection equations, Inverse Problems 33 (2017), no. 5, 055013, 22 pp.

[14] H. Jiang, F. Liu, I. Turner and K. Burrage, Analytical solutions for the multi-term time-space Caputo-Riesz fractional advection-diffusion equations on a finite domain, J. Math. Anal. Appl. 389 (2012), no. 2, 1117-1127.

[15] B. Jin, R. Lazarov and Z. Zhou, Error estimates for a semidiscrete finite element method for fractional order parabolic equations, SIAM J. Numer. Anal. 51 (2013), no. 1, 445-466. 
[16] T. Kato, Perturbation Theory for Linear Operators, Springer Science \& Business Media, 2013.

[17] Z. Li, X. Huang and M. Yamamoto, Initial-boundary value problems for multi-term time-fractional diffusion equations with $x$-dependent coefficients, Evol. Equ. Control Theory 9 (2020), no. 1, 153-179.

[18] Z. Li, Y. Liu and M. Yamamoto, Initial-boundary value problems for multi-term timefractional diffusion equations with positive constant coefficients, Appl. Math. Comput. 257 (2015), 381-397.

[19] F. Liu, P. Zhuang, V. Anh, I. Turner and K. Burrage, Stability and convergence of the difference methods for the space-time fractional advection-diffusion equation, Appl. Math. Comput. 191 (2007), no. 1, 12-20.

[20] Y. Liu, Strong maximum principle for multi-term time-fractional diffusion equations and its application to an inverse source problem, Comput. Math. Appl. 73 (2017), no. $1,96-108$.

[21] Y. Luchko, Boundary value problems for the generalized time-fractional diffusion equation of distributed order, Fract. Calc. Appl. Anal. 12 (2009), no. 4, 409-422.

[22] __ Some uniqueness and existence results for the initial-boundary-value problems for the generalized time-fractional diffusion equation, Comput. Math. Appl. 59 (2010), no. $5,1766-1772$.

[23] Initial-boundary-value problems for the generalized multi-term time-fractional diffusion equation, J. Math. Anal. Appl. 374 (2011), no. 2, 538-548.

[24] M. M. Meerschaert and C. Tadjeran, Finite difference approximations for fractional advection-dispersion flow equations, J. Comput. Appl. Math. 172 (2004), no. 1, 65-77.

[25] I. Podlubny, Fractional Differential Equations: An introduction to fractional derivatives, fractional differential equations, to methods of their solution and some of their applications, Mathematics in Science and Engineering 198, Academic Press, San Diego, 1999.

[26] K. Sakamoto and M. Yamamoto, Initial value/boundary value problems for fractional diffusion-wave equations and applications to some inverse problems, J. Math. Anal. Appl. 382 (2011), no. 1, 426-447.

[27] _ Inverse source problem with a final overdetermination for a fractional diffusion equation, Math. Control Relat. Fields 1 (2011), no. 4, 509-518. 


\section{Zhiyuan Li}

School of Mathematics and Statistics, Shandong University of Technology, Zibo, Shangdong 255049, China

E-mail address: zyli@sdut.edu.cn

Xing Cheng

College of Science, Hohai University, Nanjing, Jiangsu 210098, China

E-mail address: chengx@hhu.edu.cn

\section{Yikan Liu}

Research Institute for Electronic Science, Hokkaido University, N127W7, Kita-Ward, Sapporo 060-0812, Japan

E-mail address: ykliu@es.hokudai.ac.jp 